\title{
Agrippa d'Aubigné, CEuvres complètes: I. Petites œuvres meslees, suivies du Recueil de vers de Monsieur d'Ayre
}

\section{Michele Mastroianni}

\section{(2) OpenEdition \\ Journals}

\section{Edizione digitale}

URL: http://journals.openedition.org/studifrancesi/35952

DOI: $10.4000 /$ studifrancesi.35952

ISSN: 2421-5856

\section{Editore}

Rosenberg \& Sellier

\section{Edizione cartacea}

Data di pubblicazione: 1 juillet 2005

Paginazione: 150

ISSN: 0039-2944

\section{Notizia bibliografica digitale}

Michele Mastroianni, «Agrippa d'Aubigné, E Euvres complètes: I. Petites œuvres meslees, suivies du Recueil de vers de Monsieur d'Ayre», Studi Francesi [Online], 145 (XLIX | I) | 2005, online dal 30 novembre 2015, consultato il 18 avril 2021. URL: http://journals.openedition.org/studifrancesi/35952 ; DOI: https://doi.org/10.4000/studifrancesi.35952

Questo documento è stato generato automaticamente il 18 avril 2021.

\section{cc) $(9)$}

Studi Francesi è distribuita con Licenza Creative Commons Attribuzione - Non commerciale - Non opere derivate 4.0 Internazionale. 


\title{
Agrippa d'Aubigné, CEuvres complètes: I. Petites œuvres meslees, suivies du Recueil de vers de Monsieur d'Ayre
}

\author{
Michele Mastroianni
}

\section{NOTIZIA}

AGRIPPA D'AUBIGNÉ, CEuvres complètes: I. Petites cuvres meslees, suivies du Recueil de vers de Monsieur d'Ayre, édition critique établie et annotée par VÉRONIQUE FERRER, Paris, Champion («Textes de la Renaissance», 79), 2004, pp. 621.

1 Sotto la direzione di J.-R. Fanlo, M.-M. Fragonard e G. Schrenk prosegue l'edizione critica dell'opera omnia di Agrippa d'Aubigné (che nei «Textes de la Renaissance» aveva già visto, nel 1995, la pubblicazione dei Tragiques a cura di J.-R. Fanlo, e, nel 1996, della Responce de Michau l'aveugle e della Replique de Michau l'aveugle sempre a cura dello stesso studioso) con quello che viene indicato come il tomo I, a cura di Véronique Ferrer. Questo volume comprende anzitutto un'opera pubblicata a Ginevra due volte, nel 1629 e nel 1630, col titolo di Petites œuvres meslees. Si tratta di un recueil che riunisce quattro testi di spiritualità - le Meditations sur les Pseaumes, L'Hercule Chrestien, i Vers mesurés, L'Hyver - la cui genesi e datazione di stesura è ricostruibile con difficoltà, cosa cui peraltro si accinge, nei limiti del possibile e con ricca documentazione, V. Ferrer nella sua introduzione. Il testo è stabilito a partire dall'esemplare del 1630 conservato alla Bibliothèque Nationale, l'apparato critico segnala le varianti delle altre versioni contenute nei manoscritti Tronchin di Ginevra o nella altre opere a stampa in cui alcuni compaiono dispersi. Attraverso un'accuratissima indagine genetica V. Ferrer elucida quella che definisce pratique additive, consistente nel fatto che Agrippa d'Aubigné «conserva raramente la forma originaria del testo, che egli ricompone, dilata cesella, pur preservando l'ispirazione iniziale» (p. 24). In particolare, per quanto concerne la sezione più estesa di queste Petites œuvres, e precisamente le sei Meditations sur les Pseaumes, viene ripercorso (pp. 24-29) il cammino delle singole meditazioni, tutte 
composte in occasioni diverse che vanno dal 1588 al 1607, ma rielaborate con addizioni e varianti, a volte ripetutamente, fino all'edizione finale del 1630. Lo stesso problema d'ordine genetico si pone per le due sillogi poetiche - i Vers mesurés e L'Hyver - di cui vengono ricostruite le traversie editoriali, in una dispersione che concerne quasi tutte le composizioni, anche in questo caso sottoposte di stampa in stampa ad un processo additivo. In questo caso, poi, la riscrittura sfocia in una vera e propria métamorphose $d u$ poème (pp. 35-38), su cui si sofferma la curatrice sottolineando il passaggio di genere da una elaborazione all'altra (ad esempio, il passaggio da composizione amorosa a composizione religiosa). Così pure, viene analizzato l'éclatement générique nelle Méditations, nell'Hercule Chrestien e nei Vers mésurés, di cui si evidenzia la disparità formale (pp. 39-60), pur studiandone in seconda istanza le linee di forza che ne assicurano l'unità (pp. 60-75). L'introduzione consacra un'attenzione particolare alla ricostruzione del pensiero religioso di questi testi, pensiero che si configura in maniera forte e costante come biblismo (pp. 75-101); infine, ricostruisce il processo per cui questi testi diventano, al momento della redazione e pubblicazione ultima, una specie di testamento di Agrippa d'Aubigné, proprio per la rete complessa che si instaura nella loro storia editoriale con i grandi momenti della militanza dell'autore (pp. 101-114). Le Petites œuvres meslees, oltre che di un apparato critico esemplare, sono accompagnate da una ricca annotazione che mette in luce le fonti antiche, bibliche e contemporanee, e chiarisce allusioni storiche e difficoltà di comprensione testuale. All'edizione delle Petites cuvres meslees si aggiunge quella del Recueil des vers de Monsieur d'Ayres, manoscritto di recente ritrovato, e in annesso alcune composizioni religiose disperse negli Archivi Tronchin. Conclude un glossario e una ricca bibliografia. Il volume pertanto si presenta come edizione critica esemplare, ma anche come monografia ampia e approfondita sul metodo di lavoro, sull'evoluzione intellettuale, e sulla spiritualità di Agrippa d'Aubigné. 